\title{
Caracterización de árboles dispersos en pasturas en fincas ganaderas del departamento de Rivas, Nicaragua
}

\author{
Characterization of dispersed trees in pastures in cattle farms \\ department of Rivas, Nicaragua
}

\author{
María Chamorro Incer ${ }^{1, \star}$, Ricardo Campos Landero ${ }^{1}$ \\ y Álvaro González Martínez ${ }^{1}$
}

\begin{abstract}
Resumen
Se caracterizaron 14 pasturas con árboles dispersos en fincas ganaderas ubicadas en 9 comunidades rurales del departamento de Rivas, Nicaragua. Se realizó un inventario de los árboles dispersos en cada pastura seleccionada identificando la entidad taxonómica de cada especie, su altura total y su diámetro a la altura del pecho (DAP $\geq 5 \mathrm{~cm}$ ), y se obtuvieron datos sobre riqueza, abundancia, densidad y área de copa de los árboles. Se encontró que el $100 \%$ de las fincas y el $97.1 \%$ de los potreros tienen pasturas con árboles dispersos. Se registraron 608 árboles dispersos pertenecientes a 21 familias, 40 géneros y 45 especies. Las especies más abundantes fueron: Myrospermun frutescens Jacq, Cordia alliodora (Ruiz \& Pav.) Oken, Guazuma ulmifolia Lam. y Tabebuia rosea (Bertol.) DC. Se obtuvo en promedio una altura total, diámetro a la altura del pecho y área de copa de 9.1 $\mathrm{m}, 19.7 \mathrm{~cm}$ y $48.2 \mathrm{~m}^{2}$ respectivamente; las especies con las mayores dimensiones en altura total, DAP y área de copa fueron: Pochota fendleri (Seem.) W.S. Alverson \& M.C. Duarte, Platymiscium parviflorum Benth y Lonchocarpus minimiflorus Donn respectivamente. Las pasturas registraron una densidad arbórea promedio de 39 árboles/ha los cuales aportan un porcentaje de sombra del $14.9 \%$.
\end{abstract}

Palabras clave: riqueza, abundancia, densidad arbórea, Fabaceae.

\footnotetext{
${ }^{1}$ Unidad de Investigación, Universidad Internacional Antonio de Valdivieso (UNIAV), Rivas, Nicaragua.
}

* Autor de Correspondencia: alejandrachamorro.inves@uniav.edu.ni 


\begin{abstract}
We characterized 14 pastures with scattered trees in cattle farms located in 9 rural communities of the department of Rivas, Nicaragua. An inventory of the scattered trees in each selected pasture was made, identifying the taxonomic entity of each species, its total height and its diameter at breast height (DAP $\geq 5 \mathrm{~cm}$ ), and data were obtained on richness, abundance, density and area. of treetops. It was found that $100 \%$ of the farms and $97.1 \%$ of the paddocks have pastures with scattered trees. There were 608 scattered trees belonging to 21 families, 40 genera and 45 species. The most abundant species were: $M y$ rospermun frutescens Jacq, Cordia alliodora (Ruiz \& Pav.) Oken, Guazuma ulmifolia Lam. and Tabebuia rosea (Bertol.) DC. A total height, diameter at breast height and crown area of $9.1 \mathrm{~m}, 19.7 \mathrm{~cm}$ and $48.2 \mathrm{~m}^{2}$ respectively were obtained; the species with the largest dimensions in total height, DAP and crown area were: Pochota fendleri (Seem.) W.S. Alverson \& M.C. Duarte, Platymiscium parviflorum Benth and Lonchocarpus minimiflorus Donn respectively. Pastures recorded an average tree density of 39 trees/ha, which provide a shade percentage of $14.9 \%$.
\end{abstract}

Key words: richness, abundance, arboreal density, Fabaceae.

\section{Introducción}

En Nicaragua, la actividad ganadera representa el principal sector exportador con una tasa de crecimiento anual del 3.8\% (BCN 2012), además constituye uno de los principales usos de la tierra, para el año 2011 se estimó que el área nacional dedicada a pasturas era de 3259 634.4 ha con un hato bovino nacional de 4136 422 animales (INIDE y MAGFOR 2011).

No obstante esto ha ocasionado incrementos de las tasas de deforestación y fragmentación de paisajes (FAO 2008), siendo la degradación de pasturas un fenómeno generalizado y se estima que entre el $49-70 \%$ se encuentran en estados avanzados de degradación (Betancourt 2006; Benavides 2013) con una tasa anual de degradación que oscila entre 10-11.9\% (Holmann 2004).

La degradación de las pasturas influye en una baja productividad de carne y leche, en este sentido Nicaragua posee los menores promedios de producción en la región Centroamérica, con $133.8 \mathrm{~kg}$ carne/animal y una producción de leche ocho veces menor en comparación con Costa Rica (FAOSTAT 2012). Adicionalmente, Betancourt (2006) plantea que cuando la degradación de las pasturas se incrementa de ligera a muy severa, disminuye el rendimiento de leche y carne/vaca entre un 7-43\% lo cual implica una pérdida de productos en la región por US\$ 3.4 millones/año.

Por otro lado, cuando la ganadería es manejada con un enfoque de sistemas silvopastoriles, se reducen los efectos negativos derivados de la implementación de la ganadería intensiva, especialmente la perdida de la biodiversidad, la degradación de pasturas y del suelo. Asimismo permite un incremento y diversificación de la productividad, la sostenibilidad de los sistemas ganaderos y la generación de servicios ambientales (Ibrahim et al. 2006).

El uso de árboles dispersos en pasturas constituyen uno de los sistemas silvopastoriles más abundantes en América Latina (Grande et al. 2009; Tolentino 2009; Esquivel et al. 2013); y América Central, particularmente en zonas productoras de ganado siendo frecuentes aquellas especies que presentan múltiples usos (Harvey et al. 2010; Andrade 2007).

Los arboles dispersos desempeñan un papel importante en la diversificación de la productividad animal y en la finca, proveen sombra que favorece la disponibilidad de biomasa hasta en un 45\% (Lamela et al. 2001), tienen un efecto positivo sobre las variables fisiológicas de los animales regulando la temperatura y frecuencia respiratoria puesto que bajo la copa de los arboles estas disminuyen entre $2-3^{\circ} \mathrm{C}$ 
y 13\% respectivamente (Osorio 2014; Pezo e Ibrahim 1998; Barragán 2015), aumentan el consumo voluntario de forraje en un $12 \%$ (Osorio 2014), incrementa la producción de leche/vaca hasta en un 13.3\% (Souza de Abreu 2002), proveen una parte importante del consumo de leña $(23.5 \%)$, postes muertos $(48 \%)$, postes vivos (12\%) y madera (14.6\%) dentro de la finca (Pérez 2006), y capturan y transfieren $P$, $\mathrm{K}, \mathrm{Mg}$ y Ca hacia el suelo (Miranda et al. 2013).

Actualmente, es escaza la información que caracteriza el recurso arbóreo de las pasturas en la zona de estudio, a pesar de que se ha encontrado una tendencia a la disminución de la riqueza y abundancia de los árboles en pasturas en el departamento de Rivas (Chica 2011). Ante esta situación se realizó este estudio con el objetivo de caracterizar la presencia de árboles dispersos en pasturas de fincas ganaderas del departamento de Rivas, Nicaragua, con el fin de generar información base para el manejo, gestión y mejora del sistema silvopastoril y ganadero.

\section{Materiales y Métodos}

\section{Ubicación del área de estudio}

La investigación se desarrolló en nueve comunidades rurales del departamento de Rivas: Río Grande, Los Horconcitos, Veracruz, El Coyolito y Las Pilas ubicadas en el municipio de Rivas; La Junta ubicada en el municipio de Tola, y El Bastón, San Antonio y La Bernardino Díaz Ochoa del municipio de San Juan del Sur. Estas localidades se encuentran a $11^{\circ} 26^{\prime} 06^{\prime \prime}$ $\mathrm{N}$ y $85^{\circ} 49^{\prime} 00^{\prime \prime} \mathrm{W}$, y una altitud entre los 100 $455 \mathrm{msnm}$ (Salas 1993), con una temperatura y precipitación promedio de $27.5^{\circ} \mathrm{C}$ y 1934.5 $\mathrm{mm}$ respectivamente (Ineter 2015). De acuerdo con la clasificación de las zonas de vida de Holdridge (1996), el Bosque Seco Tropical (BST) es el tipo de vegetación dominante en el departamento de Rivas.

\section{Selección de fincas}

En las nueve comunidades se aplicó una encuesta descriptiva a un total de 69 productores para conocer el número y área de las pasturas o número de linderos y longitud con presencia de sistemas agroforestales en las fincas. La información recopilada se sistematizó en el programa estadístico SPSS versión 19. Como resultado el $71 \%$ (49 productores) tenían una o más pasturas con árboles dispersos en sus finca, para la selección de fincas, se aplicó la fórmula del tamaño de la muestra propuesta por Munch y Ángeles (1990), seleccionándose 14 fincas (una pastura a evaluar por finca) para el estudio con un área de muestreo de 19.8 ha.

\section{Caracterización de árboles dispersos en pas- turas}

Inicialmente se realizó un recorrido general por cada finca para conocer la cobertura de pastos y cantidad de árboles dispersos en las pasturas. Posteriormente se seleccionó al azar la pastura con árboles dispersos ha evaluar. En cada pastura se determinó el área y el porcentaje de inclinación del terreno, utilizando para ello un GPS MAP Garmin 78 y clinómetro Suunto, respectivamente.

Se realizó un inventario de los árboles dispersos en las pasturas, identificando y clasificando la especie de cada uno de los árboles a través de la observación directa en campo, toma de fotografías y revisión de bases de datos como MOBOT (2018), INBio (2010) e INAFOR (2009). Se contabilizó el número de individuos de cada especie, y se midió la altura total de cada individuo mediante el uso del clinómetro Suunto con base en la metodología de Chave (2005). Se estimó el área de copa de cada individuo mediante la medición perpendicular de dos diámetros de copa, el primero en dirección Este-Oeste y el segundo Norte-Sur, posteriormente se aplicó la formula expuesta por Chavarría et al. (2011). Las especies registradas fueron clasificadas según Barrance et al. (2003) en diez usos potenciales: aserrío, construcción, postes, leña, muebles, herramientas, forraje, frutos, medicinal y artesanías.

Asimismo, se estimó la abundancia de las especies mediante la frecuencia relativa, riqueza mediante el número de familias, géneros y especies encontradas, densidad arbórea mediante el número de individuos presentes en el área (Mostacedo y Fredericksen 2000) y el porcentaje de sombra se determinó según la metodología de Chavarría et al. (2001). 


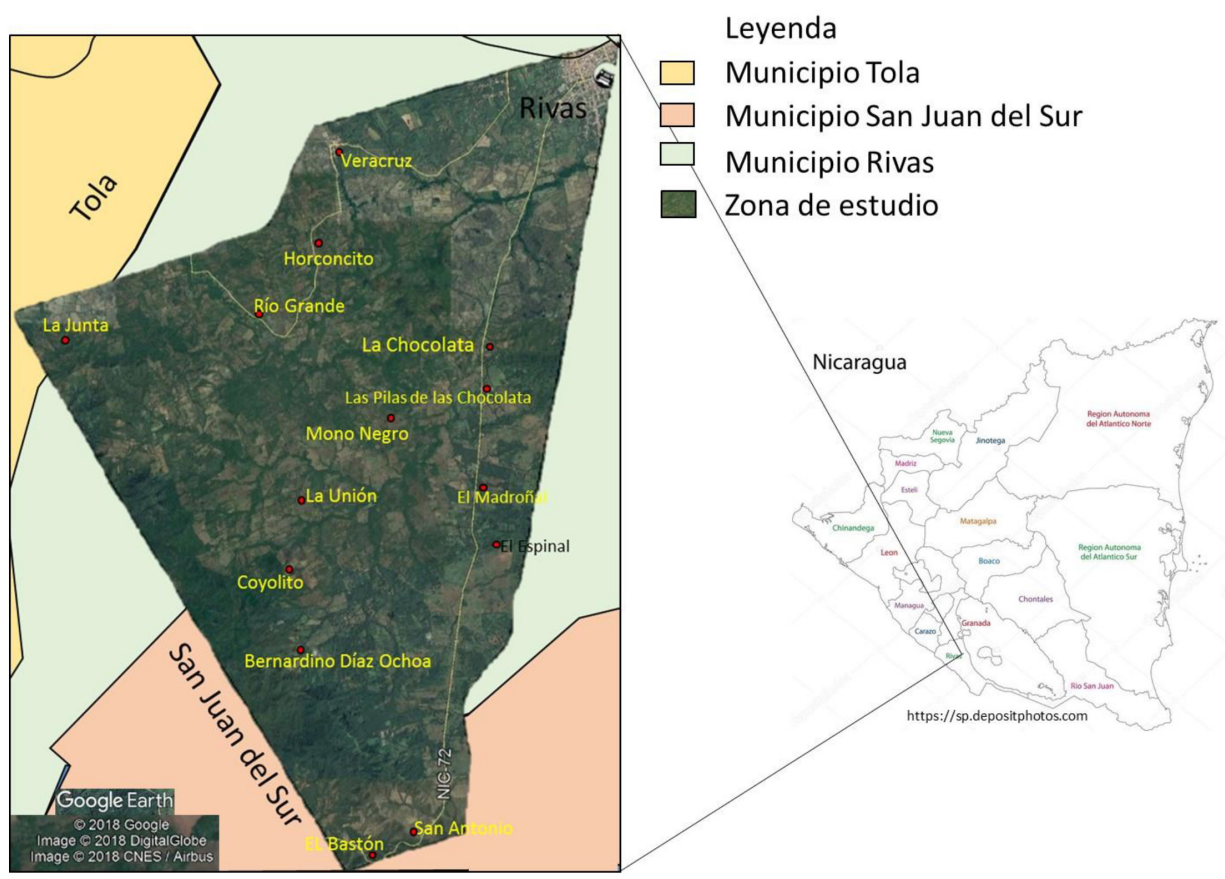

Figura 1. Mapa de localización de la zona de estudio.

\section{Resultados}

\section{Riqueza y abundancia de árboles dispersos en pasturas}

Se registró un total de 608 árboles dispersos en las pasturas pertenecientes a 21 familias, 40 géneros y 45 especies. Las cuatro familias más diversas fueron: Fabaceae (45.6\%), Bignoniaceae (14.6), Boraginaceae (13.7) y Malvaceae (11\%). Las especies más abundantes encontradas fueron Myrospermum frutescens Jacq. (Fabaceae) con 16.3\%, Cordia alliodora (Ruiz \& Pav.) Oken (Boraginaceae) con 11.7\%, Guazuma ulmifolia Lam. (Malvaceae) con 10.7\%, Tabebuia rosea (Bertol.) DC. (Bignoniaceae) con $8.7 \%$ y Diphysa americana (Mill.) M. Sousa (Fabaceae) con $8.1 \%$; la abundancia relativa de las demás especies osciló entre $0.2-0.7 \%$ (Cuadro 1).

Los cinco usos principales de las 45 especies registradas son: medicinal $(64.4 \%)$, leña (55.6\%), postes $(51.1 \%)$, construcción $(48.9 \%)$ y aserríos (46.7\%). El 48.8\% de estas especies tienen entre cinco hasta diez usos potenciales, cualidad deseable de toda especie para ser promovida y establecida en las fincas ganaderas con enfoque de sistemas. Las especies con más de ocho usos fueron Cassia grandis L.f., Spondias mombin L., Guazuma ulmifolia, Handroanthus ochraceus (Cham.) Mattos y Tabebuia rosea. Del total de especies solo de dos se desconoce su uso: Cassia moschata Kunth y Thevetia gaumeri Hemsl. (Figura 2).

En el $100 \%$ de las fincas evaluadas y en el 97.1\% de las pasturas se encontró arboles dispersos. En promedio el tamaño de las pasturas osciló entre $0.7-2.8$ ha. Se encontró que la densidad arbórea promedio por pastura fue de 39 árboles/ha, los cuales aportan un porcentaje de sombra del 14.9\% (Cuadro 2).

En relación al número de árboles por pastura, el $35.7 \%$ de estas presentaron una densidad arbórea entre 5-14 árboles/ha con un porcentaje promedio de cobertura del $1.4-4.4 \%$; el 


\begin{tabular}{|c|c|c|c|}
\hline \multirow[b]{2}{*}{ Nombre científico } & \multirow[b]{2}{*}{ Familia } & \multicolumn{2}{|c|}{ Frecuencia } \\
\hline & & Absoluta & $\begin{array}{c}\text { Relativa } \\
(\%)\end{array}$ \\
\hline Vachellia hindsii (Benth.) Seigler \& Ebinger & Fabaceae & 4 & 0.7 \\
\hline Acoelorrhaphe wrightii (Griseb. \& H.Wendl.) H.Wendl. ex Becc. & Arecaceae & 1 & 0.2 \\
\hline Bauhinia purpurea $\mathrm{L}$. & Fabaceae & 1 & 0.2 \\
\hline Bursera simarouba (L.) Sarg. & Burseraceae & 1 & 0.2 \\
\hline Bursera graveolens (Kunth) Triana \& Planch. & Burseraceae & 1 & 0.2 \\
\hline Byrsonima crassifolia (L.) Kunth & Malpighiaceae & 11 & 1.8 \\
\hline Calycophyllum candidissimum (Vahl) DC. & Rubiaceae & 2 & 0.3 \\
\hline Cassia grandis L.f., Suppl. & Fabaceae & 4 & 0.7 \\
\hline Cassia moschata Kunth & Fabaceae & 1 & 0.2 \\
\hline Cedrela odorata L. & Meliaceae & 3 & 0.5 \\
\hline Chomelia spinosa Jacq. & Rubiaceae & 2 & 0.3 \\
\hline Citrus aurantium $\mathrm{L}$. & Rutaceae & 1 & 0.2 \\
\hline Coccoloba caracasana Meisn. & Polygonaceae & 8 & 1.3 \\
\hline Cochlospermum vitifolium (Willd.) Spreng. & Bixaceae & 5 & 0.8 \\
\hline Cordia alliodora (Ruiz \& Pav.) Oken & Boraginaceae & 71 & 11.7 \\
\hline Cordia bicolor A. DC. & Boraginaceae & 3 & 0.5 \\
\hline Cordia dentata Poir. & Boraginaceae & 12 & 2.0 \\
\hline Crescentia alata Kunth & Bignoniaceae & 13 & 2.1 \\
\hline Diospyros nicaraguensis (Standl.) Standl. & Ebenaceae & 2 & 0.3 \\
\hline Diphysa americana (Mill.) M. Sousa & Fabaceae & 49 & 8.1 \\
\hline Enterolobium cyclocarpum (Jacq.) Griseb. & Fabaceae & 13 & 2.1 \\
\hline Erythrina fusca Lour. & Fabaceae & 10 & 1.6 \\
\hline Erythrostemon exostemma (DC.) Gagnon \& G.P. Lewis & Fabaceae & 18 & 3.0 \\
\hline Gliricidia sepium (Jacq.) Kunth ex Walp. & Fabaceae & 45 & 7.4 \\
\hline Guazuma ulmifolia Lam. & Malvaceae & 65 & 10.7 \\
\hline Handroanthus ochraceus (Cham.) Mattos & Bignoniaceae & 23 & 3.8 \\
\hline Karwinskia calderonii Standl. & Rhamnaceae & 17 & 2.8 \\
\hline Lonchocarpus costaricensis (Donn. Sm.) Pittier & Fabaceae & 18 & 3.0 \\
\hline Lonchocarpus miniflorus Donn. Sm. & Fabaceae & 6 & 1.0 \\
\hline Myrospermum frutescens Jacq. & Fabaceae & 99 & 16.3 \\
\hline Platymiscium parviflorum Benth. & Fabaceae & 1 & 0.2 \\
\hline Pochota fendleri (Seem.) W.S. Alverson \& M.C. Duarte & Malvaceae & 2 & 0.3 \\
\hline Pseudosamanea guachapele (Kunth) Harms & Fabaceae & 3 & 0.5 \\
\hline Psidium guajava L. & Myrtaceae & 1 & 0.2 \\
\hline
\end{tabular}

Cuadro 1. Riqueza y abundancia de especies arbóreas registradas en pasturas con árboles dispersos de fincas ganaderas del departamento de Rivas, Nicaragua (continua en la siguiente página). 

del departamento de Rivas, Nicaragua

\begin{tabular}{|c|c|c|c|}
\hline \multirow[b]{2}{*}{ Nombre científico } & \multirow[b]{2}{*}{ Familia } & \multicolumn{2}{|c|}{ Frecuencia } \\
\hline & & Absoluta & $\begin{array}{c}\text { Relativa } \\
(\%)\end{array}$ \\
\hline Samanea saman (Jacq.) Merr. & Fabaceae & 5 & 0.8 \\
\hline Sapranthus nicaraguensis Seem. & Annonaceae & 1 & 0.2 \\
\hline Sideroxylon capiri (A. DC.) Pittier & Sapotaceae & 3 & 0.5 \\
\hline Simarouba glauca DC. & Simaroubaceae & 4 & 0.7 \\
\hline Spondias mombin L. & Anacardiaceae & 4 & 0.7 \\
\hline Spondias purpurea $\mathrm{L}$. & Anacardiaceae & 15 & 2.5 \\
\hline Stemmadenia obovata K. Schum. & Apocynaceae & 1 & 0.2 \\
\hline Swietenia humilis Zucc. & Meliaceae & 4 & 0.7 \\
\hline Tabebuia rosea (Bertol.) DC. & Bignoniaceae & 53 & 8.7 \\
\hline Terminalia oblonga (Ruiz \& Pav.) Steud. & Combretaceae & 1 & 0.2 \\
\hline Thevetia gaumeri Hemsl. & Apocynaceae & 1 & 0.2 \\
\hline Total & 21 & 608 & 100 \\
\hline
\end{tabular}

Cuadro 1. Riqueza y abundancia de especies arbóreas registradas en pasturas con árboles dispersos de fincas ganaderas del departamento de Rivas, Nicaragua (viene de la página anterior).

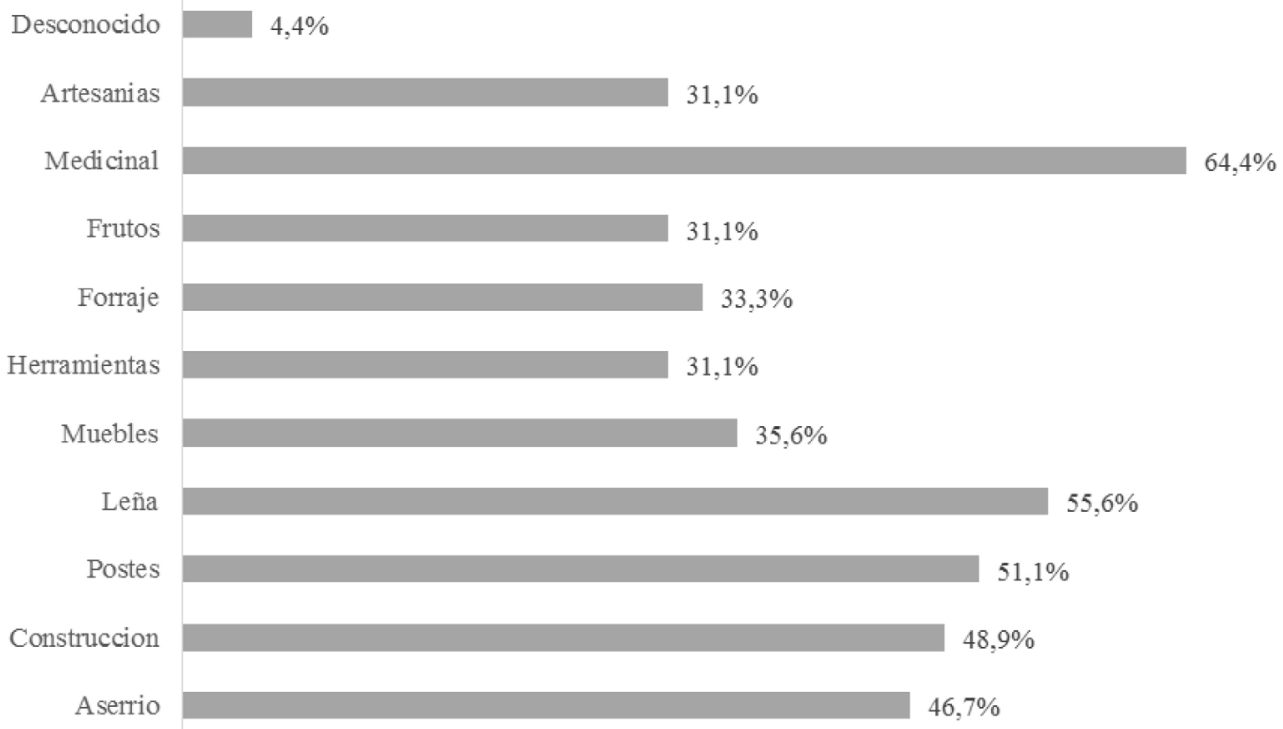

Figura 2. Porcentaje de especies registradas con usos en árboles dispersos en pasturas de fincas ganaderas del departamento de Rivas, Nicaragua. 


\begin{tabular}{|c|c|c|c|c|c|}
\hline Finca & $\begin{array}{c}\mathbf{N}^{\mathbf{0}} \text { de pasturas con } \\
\text { árboles dispersos/ } \\
\text { finca }\end{array}$ & Área (ha) & $\mathbf{N}^{\mathbf{0}}$ árboles & $\mathbf{N}^{\mathbf{0}}$ árboles/ha & $\begin{array}{c}\text { Porcentaje } \\
\text { de sombra/ } \\
\text { pastura }\end{array}$ \\
\hline Buena Vista & 5 & 1.2 & 31 & 26 & 10.9 \\
\hline El Carmen & 13 & 2.8 & 65 & 23 & 11.1 \\
\hline San José & 12 & 1.4 & 27 & 20 & 10.2 \\
\hline Dolores & 1 & 0.7 & 86 & 128 & 35.9 \\
\hline El Mango & 5 & 1.6 & 13 & 8 & 4.4 \\
\hline El Neem & 4 & 1.5 & 7 & 5 & 1.4 \\
\hline El Nambaro & 3 & 1.2 & 159 & 133 & 40.1 \\
\hline El Pensamiento & 3 & 0.9 & 23 & 25 & 39.2 \\
\hline Las 2 G & 3 & 0.7 & 39 & 56 & 14.0 \\
\hline San Pedro & 6 & 2.8 & 15 & 5 & 1.6 \\
\hline Los Ángeles & 4 & 0.9 & 5 & 6 & 3.2 \\
\hline Santa Elena & 8 & 0.9 & 69 & 73 & 17.6 \\
\hline San Antonio & 3 & 1.1 & 15 & 14 & 4.4 \\
\hline Santa Marta & 14 & 2.1 & 54 & 25 & 14.8 \\
\hline Promedio & $\mathbf{8 4}$ & $\mathbf{1 . 4}$ & $\mathbf{4 3 . 4}$ & $\mathbf{3 9 . 0}$ & $\mathbf{1 4 . 9}$ \\
\hline
\end{tabular}

Cuadro 2. Numero de pasturas con árboles dispersos/finca, área, densidad arbórea y porcentaje de sombra/pastura en fincas ganaderas del departamento de Rivas, Nicaragua.

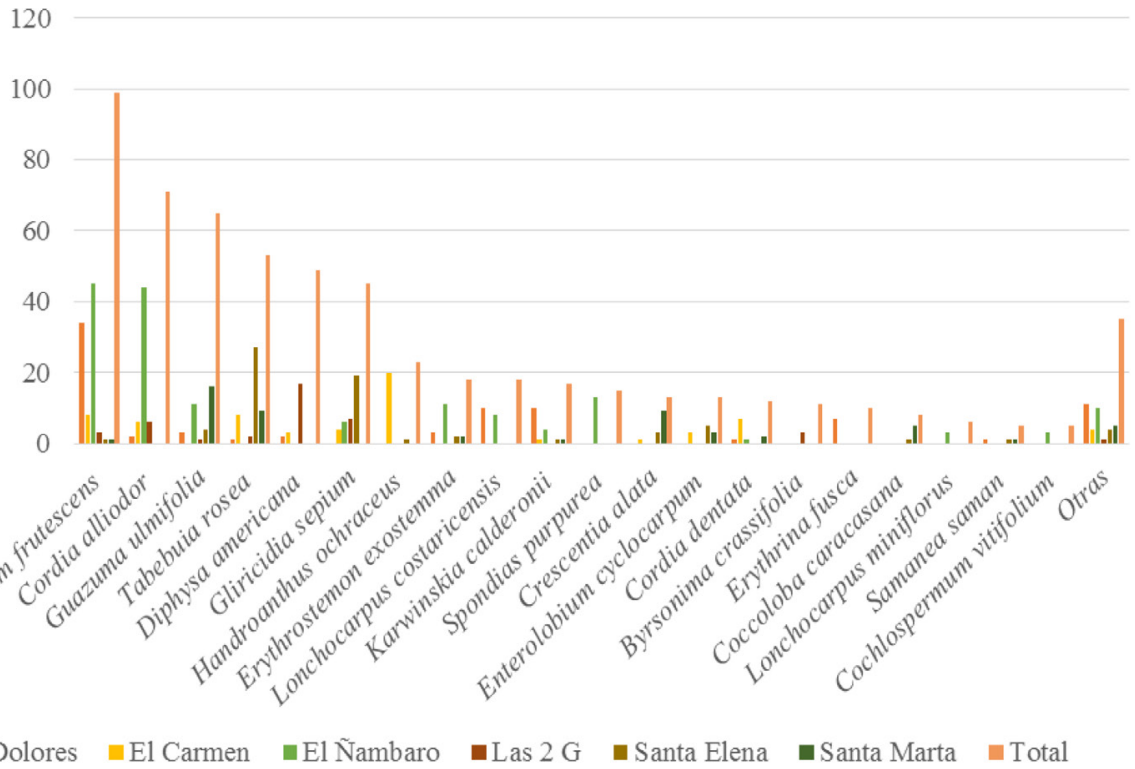

Figura 3. Fincas con la mayor abundancia de especies de árboles dispersos en pasturas del departamento de Rivas, Nicaragua. 

del departamento de Rivas, Nicaragua

42.9\% entre 20 a 73 árboles/ha con un porcentaje de sombra que osciló entre 10.2-17.6\%, y el $21.4 \%$ entre $25-128$ árboles/ha y un porcentaje de cobertura entre $35.9-40.1 \%$ (Cuadro 2).

Las fincas con la mayor abundancia de especies fueron: El Ñambaro, Dolores, Santa Elena, El Carmen y Santa Marta. La especie Myrospermum frutescens fue la más representativa en la finca El Nambaro y Dolores, con un 28.3 y $39.5 \%$ respectivamente, así mismo, en la finca el Nambaro las especies Cordia alliodora y Guazuma ulmifolia también fueron importantes con un 27.7 y $8.8 \%$, respectivamente. En las fincas Santa Elena y Las 2 G, Tabebuia rosea y Diphysa americana son las especies con mayor abundancia con el 39.1 y $42.5 \%$, respectivamente (Figura 3).

Las especies Psidium guajava L. (El Pensamiento), Stemmadenia obovata K. Schum., Sapranthus nicaraguensis Seem. y Bauhinia purpurea L. (El Nambaro), Bursera simarou- ba (L.) Sarg., Citrus aurantium L. y Cassia moschata (Santa Elena), Thevetia gaumeri Hemsl. (Dolores), Platymiscium parviflorum Benth. y Bursera graveolens (Kunth) Triana \& Planch. (El Carmen), Terminalia oblonga (Ruiz \& Pav.) Steud. (San José) y Vachellia hindsii (Benth.) Seigler \& Ebinger (Las 2 G); son las especies que registraron la menor abundancia con el 1.9\% (12 especies) en seis de las 14 fincas (Cuadro 3).

Los árboles dispersos presentaron una altura total, DAP y área de copa promedio de $9.1 \mathrm{~m}$, $19.7 \mathrm{~cm}$ y $48.2 \mathrm{~m}^{2}$ respectivamente. Las especies Pochota fendleri (Seem.) W.S. Alverson \& M.C. Duarte, Platymiscium parviflorum y Lonchocarpus miniflorus Donn. Sm. registraron las mayores dimensiones en altura total $(26.6 \mathrm{~m})$, DAP $(54 \mathrm{~cm})$ y área de copa $\left(250 \mathrm{~m}^{2}\right)$ respectivamente. Se encontró que más del $37 \%$ de las especies registraron una altura total y DAP superior al promedio, y el 35.6\% un área de copa superior al promedio (Cuadro 3 ).

\begin{tabular}{|c|c|c|c|}
\hline Especie & $\begin{array}{l}\text { Altura total } \\
\text { (m) }\end{array}$ & DAP (cm) & $\mathrm{AC}\left(\mathbf{m}^{2}\right)$ \\
\hline Vachellia hindsii (Benth.) Seigler \& Ebinger & 6.2 & 11.9 & 14.8 \\
\hline Acoelorrhaphe wrightii (Griseb. \& H.Wendl.) H.Wendl. ex Becc. & 8.7 & 39.0 & 4.9 \\
\hline Bauhinia purpurea $\mathrm{L}$. & 8.5 & 7.1 & 7.1 \\
\hline Bursea simarouba (L.) Sarg. & 5.7 & 7.4 & 6.3 \\
\hline Bursera graveolens (Kunth) Triana \& Planch. & 8.0 & 21.5 & 40.6 \\
\hline Byrsonima crassifolia (L.) Kunth & 8.9 & 18.5 & 41.2 \\
\hline Calycophyllum candidissimum (Vahl) DC. & 8.8 & 19.2 & 90.4 \\
\hline Cassia grandis L. f., Suppl. & 8.6 & 20.9 & 87.8 \\
\hline Cassia moschata Kunth & 8.0 & 11.9 & 35.2 \\
\hline Cedrela odorata $\mathrm{L}$. & 10.0 & 14.3 & 13.0 \\
\hline Chomelia spinosa Jacq. & 5.8 & 10.1 & 26.1 \\
\hline Citrus aurantium $\mathrm{L}$. & 5.3 & 6.7 & 11.4 \\
\hline Coccoloba caracasana Meisn. & 8.3 & 26.0 & 48.1 \\
\hline Cochlospermum vitifolium (Willd.) Spreng. & 10.2 & 25.4 & 39.3 \\
\hline Cordia alliodora (Ruiz \& Pav.) Oken & 10.8 & 19.3 & 28.9 \\
\hline
\end{tabular}

Cuadro 3. Altura total, diámetro a la altura del pecho (DAP) y área de cobertura (AC) de árboles dispersos en fincas ganaderas del departamento de Rivas, Nicaragua (continua en la siguiente página anterior). 


\begin{tabular}{|c|c|c|c|}
\hline Especie & $\begin{array}{l}\text { Altura total } \\
\text { (m) }\end{array}$ & DAP (cm) & $\operatorname{AC}\left(m^{2}\right)$ \\
\hline Cordia bicolor A. DC. & 8.4 & 26.7 & 66.3 \\
\hline Cordia dentata Poir. & 9.2 & 24.4 & 51.8 \\
\hline Crescentia alata Kunth & 8.3 & 27.0 & 57.7 \\
\hline Diospyros nicaraguensis (Standl.)Standl. & 7.9 & 14.1 & 23.2 \\
\hline Diphysa americana (Mill.)M. Sousa & 9.0 & 18.8 & 28.7 \\
\hline Enterolobium cyclocarpum (Jacq.)Griseb. & 12.8 & 32.3 & 104.6 \\
\hline Erythrina fusca Lour. & 8.5 & 16.1 & 31.6 \\
\hline Erythrostemon exostemma (DC.) Gagnon \& G.P. Lewis & 9.2 & 16.2 & 27.1 \\
\hline Gliricidia sepium (Jacq.) Kunth ex Walp. & 9.7 & 15.4 & 25.7 \\
\hline Guazuma ulmifolia Lam. & 11.2 & 30.3 & 69.6 \\
\hline Handroanthus ochraceus (Cham.) Mattos & 9.3 & 22.5 & 35.9 \\
\hline Karwinskia calderonii Standl. & 7.5 & 17.5 & 39.0 \\
\hline Lonchocarpus costaricensis (Donn. Sm.) Pittier & 7.3 & 10.9 & 18.4 \\
\hline Lonchocarpus miniflorus Donn. Sm. & 13.1 & 28.7 & 250.0 \\
\hline Myrospermum frutescens Jacq. & 8.0 & 11.6 & 22.3 \\
\hline Platymiscium parviflorum Benth. & 9.3 & 54.0 & 116.9 \\
\hline Pochota fendleri (Seem.) W.S. Alverson \& M.C. Duarte & 26.6 & 39.0 & 119.8 \\
\hline Pseudosamanea guachapele (Kunth) Harms & 10.8 & 34.8 & 79.6 \\
\hline Psidium guajava $\mathrm{L}$. & 6.2 & 11.5 & 24.4 \\
\hline Samanea aman (Jacq.) Merr. & 12.0 & 14.8 & 100.2 \\
\hline Sapranthus nicaraguensis Seem. & 7.0 & 9.4 & 17.4 \\
\hline Sideroxylon capiri (A. DC.) Pittier & 12.9 & 17.5 & 109.5 \\
\hline Simarouba glauca DC. & 6.4 & 14.4 & 19.4 \\
\hline Spondias mombin L. & 9.2 & 35.9 & 84.5 \\
\hline Spondias purpurea L. & 7.1 & 21.8 & 43.4 \\
\hline Stemmadenia obovata K. Schum. & 7.0 & 13.5 & 16.3 \\
\hline Swietenia humilis Zucc. & 7.6 & 7.9 & 9.4 \\
\hline Tabebuia rosea (Bertol.) DC. & 8.1 & 17.3 & 25.2 \\
\hline Terminalia oblonga (Ruiz \& Pav.) Steud. & 7.6 & 10.8 & 17.4 \\
\hline Thevetia gaumeri Hemsl. & 9.9 & 10.3 & 36.3 \\
\hline Promedio & 9.1 & 19.7 & 48.2 \\
\hline
\end{tabular}

Cuadro 3. Altura total, diámetro a la altura del pecho (DAP) y área de cobertura (AC) de árboles dispersos en fincas ganaderas del departamento de Rivas, Nicaragua (viene de la página anterior). 

del departamento de Rivas, Nicaragua

\section{Discusión}

En las pasturas con árboles dispersos evaluadas se registraron 608 individuos, 21 familias y 45 especies en un área de muestreo de 19.8 ha, esto refleja una riqueza y abundancia menor en más del $20 \%$ a los estimados en fincas ganaderas de Belén (Rivas, Nicaragua) donde se registraron 840 individuos, 27 familias y 63 especies en un área de muestreo de 22.1 ha (Chica 2011). Las diferencias en los valores de riqueza se deben posiblemente a un menor área de muestreo, no obstante a pesar de encontrar estas diferencias, la familia Fabaceae fue la más diversa, debido a que ambas zonas se ubican en el departamento de Rivas y por lo tanto poseen la misma altitud, siendo este el parámetro climático con más peso discriminante en la determinación de la riqueza en los tipos de bosque (Murrieta et al. 2007); asimismo es la tercera familia más diversa de las zonas tropicales y húmedas (Wojciechowski et al. 2004), y se encuentra ampliamente distribuida a nivel Centroamericano (López et al. 2015; Mora et al. 2015). Por otra parte es importante destacar que los productores del departamento de Rivas tienen un conocimiento profundo acerca de la importancia, usos, relaciones y efectos acerca la conservación de los arboles dispersos en las pasturas, que se ha fomentado a través de la concientización e implementación de proyectos que permitan su conservación entre ellos destaca el Proyecto FRAGMEN (Joya et al. 2004).

Las especies arbóreas Myrospermum frutescens, Cordia alliodora, Guazuma ulmifolia y Tabebuia rosea fueron las más abundantes, esto se debe a que estas especies son típicas de áreas perturbadas y comúnmente próximas a bosques secundarios, registran un alto índice de valor de importancia y sus semillas son dispersadas por medio del viento, animales silvestres y el ganado; lo cual las convierte en especies pioneras en la regeneración natural (Esquivel et al. 2009); estos resultados coinciden con lo determinado por Esquivel et al. (2003) en Guanacaste (Costa Rica), donde el 37.4\% del total de individuos están representados por tres especies: C. alliodora (12\%), G. ulmifolia (12.6\%) y T. rosea (12.8\%). La especie G. ulmifolia posee una amplia distribución en los potreros de fincas ganaderas de Centroamérica y América del Sur. Serrano et al. (2014), reportan que esta especie representó el $25 \%$ del total de individuos inventariados en potreros del bosque seco en Tolima (Colombia).

Según Esquivel et al. (2003) en Guanacaste (Costa Rica) las especies menos abundantes fueron: Bursera simarouba, Samanea saman, Enterolobium cyclocarpum, Cedrela odorata y Spondias purpurea con valores de 2.2, 1.3, 0.9, 1.1 y $1.4 \%$ respectivamente. Lo que coincide con lo encontrado en este estudio, puesto que la abundancia de estas especies oscilo entre $0.2-2.1 \%$; esto puede explicarse debido a que los productores llevan a cabo actividades de manejo en las pasturas como: como control de arvenses (seleccionando los arbustos que dejaran o eliminaran), eliminando así individuos en estadios juveniles, afectando así la regeneración natural de las especies (Harvey et al. 2007).

En relación al uso, el 33.3\% de las 45 especies tienen uso forrajero y el $46.7 \%$ para aserríos, superior a lo encontrado por Chica (2011), quien estimo que el $15.2 \%$ tienen uso forrajero y $19.6 \%$ maderable (aserríos); en ambos estudios se evidencia que los productores prefieren mantener en sus potreros especies con uso forrajero que aporten alimento para el ganado (follaje y frutos) y de uso maderable que generen productos visibles para uso interno en la finca. Entre las especies de uso forrajero que destacan en ambos estudios están: Cassia grandis, Guazuma ulmifolia y Spondias mombin y entre las de uso maderable: Cordia dentata Poir., Gliricidia sepium (Jacq.) Kunth ex Walp., Calycophyllum candidissimum (Vahl) DC. y Diphysa americana (Joya et al. 2004; Chica 2011).

El área de las pasturas de las 14 fincas oscilo entre 0.7-2.8 ha, menor a los 0.1-39.5 ha de las pasturas en fincas ganaderas de Guanacaste (Costa Rica) estimados por Esquivel et al. (2003), estas discrepancias se deben probablemente a las diferencias en la tipología de finca entre ambas localidades puesto que en el departamento de Rivas el $48.2 \%$ de las fincas son manejadas con poca tecnificación (Chica 2011). 
La cantidad promedio de árboles por pastura de una ha fue de 39, esto difiere a lo reportado por Pérez (2006) en Copán (Honduras) y Sánchez et al. (2003) en Belén (Rivas, Nicaragua), quienes estimaron un promedio de 55 y 16.2 árboles/ha respectivamente. En promedio el porcentaje de sombra en las pasturas (14.9\%), es considerado moderado y tiene un efecto positivo sobre la disponibilidad de biomasa del pasto principalmente en la época de sequía (Esquivel 2007); este resultado contrasta con los $42.9 \%$ estimados por Chavarría et al. (2011) en Copán (Honduras); y es similar al 13\% estimado por Chica (2011), en fincas ganaderas de Belén (Rivas, Nicaragua).

La altura total promedio de los arboles fue de $9.1 \mathrm{~m}$, superior a los $8.7 \mathrm{~m}$ estimados por Chica (2011) en Belén (Rivas, Nicaragua) e inferior a los $24.2 \mathrm{~m}$ estimado por Harvey et al. (2007) Rivas (Nicaragua); en lo referido al DAP y el área de copa los datos promedios corresponden a $19.7 \mathrm{~cm}$ y $48.2 \mathrm{~m}^{2}$, difieren a los amplios rangos de $8.0-36.8 \mathrm{~cm}$ de DAP y $12.2-87.6 \mathrm{~m}^{2}$ en área de copa, estimados por Harvey et al. (2007) en Rivas (Nicaragua); estas discrepancias se deben a las amplias diferencias en el área de muestro y la cantidad de árboles dispersos inventariados, superior en más $250 \%$ en comparación con este estudio.

Asimismo, Esquivel et al. (2003) reportan que las mayores dimensiones en altura total lo presentaron las especies: Enterolobium cyclocarpum (Jacq.) Griseb. (15.9 m), Samanea saman (Jacq.) Merr. (14.2 m), Cordia alliodora (13.4 m), Guazuma ulmifolia (10.8 m), y Byrsonima crassifolia (L.) Kunth ( $8.6 \mathrm{~m}$ ), esto coincide a lo encontrado en este estudio puesto que estas especies registraron los siguientes valores: $12.8 \mathrm{~m}, 12 \mathrm{~m}, 10.8 \mathrm{~m}, 11.2 \mathrm{~m}$ y $8.9 \mathrm{~m}$ respectivamente.

\section{Conclusiones}

En los ecosistemas ganaderos estudiados aún se mantiene la diversidad florística y biológica con el uso de especies multifuncionales, el $100 \%$ de las fincas tienen pasturas con árboles dispersos y entre el $66.7-100 \%$ de las pasturas de cada finca contienen árboles.
Las especies Pseudosamanea guachapele (Kunth) Harms, Bursera simarouba (L.) Sarg, Coccoloba caracasana Meisn, Cassia grandis L.f., Suppl, Cordia dentata Poir, Diphysa americana (Mill.) M. Sousa y Guazuma ulmifolia Lam, encontradas como árboles dispersos, son especies multifuncionales, es decir proporcionan diversos usos dentro de la finca como: leña, postes, construcción, aserríos, medicinales, etc.

El componente arbóreo según Louman et al. (2002), se encuentra en la etapa de fustal $(\mathrm{DAP}>10 \mathrm{~cm})$, con una mezcla diversa de especies con diferentes características, dimensiones, tamaños y formas de copa. Además, el porcentaje de sombra de los árboles en las pasturas es moderado $(<15 \%)$ y con una densidad arbórea de 39 árboles/ha, lo que favorece la cobertura y disponibilidad de biomasa de pasto.

\section{Agradecimientos}

A la Universidad Internacional Antonio de Valdivieso (UNIAV), a su Rector Carlos Írias Amaya y a su Vicerrector Académico Douglas Marín Briones por aprobar y apoyar la ejecución del Proyecto de Investigación Caracterización de Sistemas Agroforestales de donde se deriva este estudio. Al M.Sc. Joel Rojas por sus recomendaciones durante todo este proceso. Al M.Sc. Francisco Chavarría, compañeros de trabajo y equipo técnico que nos acompañó por su compromiso en el levantamiento de la información. A los productores del municipio de Rivas, Tola y San Juan del Sur por su colaboración.

\section{Bibliografía}

Andrade, H; Brook, R; Ibrahim, M. 2008. Growth, production and carbon sequestration of silvopastoral systems with native timber species in the dry lowlands of Costa Rica (en línea) Plaint Soil 308: 11-22. Consultado 12 set. 2017. DOI: http://dx.doi.org/10.1007/s11104008-9600-x

Barragán, W; Mahecha; Cajas, Y. 2015. Variables fisiológicas-metabólicas de estrés calórico en vacas bajo silvopastoreo y pradera sin ár- 
boles (en línea). Agronomía Mesoamericana. 26(2): 211-223. Consultado 23 ene. 2017. Disponible en https://revistas.ucr.ac.cr/index.php/ agromeso/article/view/19277/19409

Barrance, A; Beer, J; Boshier, J; Chamberlain, J, Cordero, J; Detlefsen, G; Finegan, B; Galloway, G; Gómez, M; Gordon, J; Hands, M; Hellin, J; Hughes, C; Ibrahim, M; Leakey R; Mesen, F; Montero, M; Montero, M; Somarriba, E; Stewart, J. Árboles de Centroamérica: Un manual para extensionistas (Disco compacto). Oxford Forestry Institute: Department of Plant Sciences, University of Oxford y Centro Agronómico Tropical de Investigación y Enseñanza, Costa Rica. 1 Disco compacto, $8 \mathrm{~mm}$, sin sonido y confección del material a color.

BCN (Banco Central de Nicaragua). 2012. Anuario Estadístico (en línea). Managua, Nicaragua. 172 p. Consultado 23 ene. 2017. Disponible en http://www.bcn.gob.ni/publicaciones/periodicidad/anual/anuario estadistico/2012/anuario estadistico 2012.pdf

Benavides, M. 2013. Evaluación del impacto socioeconómico de pasturas degradadas en fincas ganaderas de la cuenca media del río Jesús Maria, Costa Rica. Tesis Mag. MSc. Turrialba, Costa Rica. CATIE. 74 p.

Betancourt, H; Pezo, D; Cruz, J; Beer, J. 2007. Impacto bioeconómico de la degradación de pasturas en fincas de doble propósito en El Chal, Petén, Guatemala (en línea). Pastos y Forrajes 30(1): 169-175. Consultado 19 abr. 2016. Disponible en http://www.redalyc.org/ pdf/2691/269119705005.pdf

Chavarría, A; Detlefsen, G; Ibrahim, M; Galloway, G; de Camino; R. 2011. Análisis de la productividad y contribución financiera del componente arbóreo en pequeñas y medianas fincas ganaderas de la subcuenca del Rio Copán, Honduras. Agroforestería de las Américas (48): 146-156.

Chave, J. 2005. Medición de la altura del árboles, para arboles tropicales. Manual de campo. Toulouse, France. Proyecto de Avance de las Redes Científicas en el Amazonia. 3 p.

Chica, DM. 2011. Análisis de la relación entre la cobertura y composición arbórea, factores de manejo y productividad en fincas ganaderas doble propósito del departamento de Rivas, Nicaragua. Tesis Mag. MSc. Turrialba, Costa Rica. CATIE. 94 p.

Esquivel, E; Ibrahim, m; Harvey, C, Villanueva, C; Benjamín, T; Sinclair; F. 2003. Árboles dispersos en fincas ganaderas en un ecosistema seco de Costa Rica. Agroforestería de las Américas 10(39-40): 24-29.

Esquivel, M. 2007. Tree resources in traditional silvopastoral systems and their impacts on productivity and nutritive value of pastures in the dry tropics of Costa Rica. Tesis Ph.D. Turrialba, Costa Rica. CATIE. Consultado 10 oct. 2018. Disponible en http://orton.catie.ac.cr/repdoc/ A1656e/A1656e.pdf

Esquivel, M; Harvey, C; Finegan, B; Cassanoves, F; Skarpe, C y Nieuwenhuyse, A. 2009. Regeneración natural de árboles y arbustos en potreros activos de Nicaragua. Agroforestería en las Américas (en línea) (47): 76-84. Consultado 16 oct. 2018. Disponible en https://www. researchgate.net/profile/Fernando Casanoves/ publication/283491193 Natural_regeneration of trees and shrubs in active pastures in Nicaragua/links/566b856508aea0892c4c794b.pdf

FAO (Food and Agricultural Organization of the United Nations). 2008. Ayudando a desarrollar una ganadería sustentable en Latinoamérica y El Caribe. Lecciones a partir de casos exitosos (en línea). Santiago. ISBN 97892-5-30597-6. Consultado 19 abr. 2016. Disponible en http://www.fao.org/tempref/docrep/fao/010/I0082s/I0082s01.pdf

FAOSTAT (Statistics Division Food and Agricultural Organization of the United Nations, Italia). 2012. Statistics the Livestock Primary and Livestock Processed in Central America (en línea). Roma, Italia. Consultado 31 ene. 2017. Disponible en http://www.fao.org/tempref/docrep/fao/010/I0082s/I0082s01.pdf

Grande, D; Losada, H; Cortes, J; Rivera, J; Maldonado, M; Pérez, F. 2009. Los Árboles Dispersos en Potreros de la Región de la Sierra de Tabasco, México (en línea). Agroecología 
4(2): 4489-4492. Consultado 26 abr. 2016. Disponible en http://www.aba-agroecologia. org.br/revistas/index.php/cad/article/viewFile/4723/3502

Harvey, C; Villanueva, C; Esquivel, H; Gómez, R; Ibrahim, M; López, M; Martínez, J; Muños, D; Claudia, R; Saénz, J; Villacis, J; Sinclaier, F. 2010. Conservation value of dispersed tree cover threatened by pasture management. Forest Ecology and Management 26 (10): 1664-1674.

Harvey, C; Villanueva, C; Ibrahim, M; Gomez, R; Lopez, M; Kunth, S y Sinclair, L. 2007. Evaluación y conservación de biodiversidad en paisajes fragmentados de Mesoamérica. Eds. Harvey, C; Sáenz, J. 1ra edición. Santo Domingo de Heredia, Costa Rica. INBio. 624 p. ISBN 978-9968-927-29-1

Holdridge, L. 1996. Ecología basada en zonas de vida. 4ta edición. IICA. 216 p. ISBN 929039-1316.

Holmann, F; Argel, P; Rivas, L; White, D; Estrada, R.D; Burgos, C; Pérez, E; Ramírez, G; Medina, A. 2004. Degradación de pasturas y pérdidas de productividad animal: Una evaluación económica desde la perspectiva de los productores y extensionistas pecuarios en Honduras. CIAT. 42 p. Consultado 8 feb. 2017 Disponible en http://ciat-library.ciat.cgiar.org/ articulos ciat/tropileche/Degradacion de pasturas.pdf

Ibrahim, M; Mora, J; Rosales, M. 2006. Potencialidades de los sistemas silvopastoriles para la generación de servicios ambientales. In Ibrahim, M; Mora, J y Rosales, M (eds.) Memorias de una conferencia electrónica "Potencialidades de los sistemas silvopastoriles para la generación de servicios ambientales". Turrialba, Costa Rica. CATIE. p 9-12.

INETER (Instituto de Estudios Territoriales). 2014. Datos meteorológicos del departamento de Rivas en el 2015 (en línea). Rivas, Nicaragua. Consultado 19 abr. 2016. Disponible en http://www.ineter.gob.ni/

INIDE (Instituto Nacional de Información de Desarrollo) y MAGFOR (Instituto Agropecuario y Forestal). 2011. Informe del IV Censo
Nacional Agropecuario (en línea). Managua, Nicaragua. 70 p. Consultado 19 abr. 2016. Disponible en http://www.inide.gob.ni/Cenagro/ INFIVCENAGRO/IVCENAGROINFORME/ assets/basic-html/page9.html

Instituto Nacional de Biodiversidad (INBio). 2010. Sistema Costarricense de Información sobre Biodiversidad (en línea). Heredia, Costa Rica. Consultado 17 set. 2018. Disponible en https://www.gbif.org/publisher/ 5c7a5c20-1bd0-11d8-a2da-b8a03c50a862

Instituto Nacional Forestal (INAFOR). 2009. Resultados del Inventario Nacional Forestal: Nicaragua 2007-2008/INAFOR (en línea). 2da edición. Managua, Nicaragua. 22 p. ISBN 97899924-0-846-9

Joya, M; López, M; Gómez, R y Harvey, C. 2004. Conocimiento local sobre el uso y manejo de los árboles en las fincas ganaderas del municipio de Belén, Rivas (en línea). Encuentro 18: 1 - 17. UCA - CATIE. Managua, Nicaragua. Consultado 3 may. 2018. Disponible en https:// www.lamjol.info/index.php/ENCUENTRO/ article/view/4256/3997

López, O; Pérez, R y Mariscal. E. 2015. Diversidad de árboles y arbustos en fragmentos de bosque seco tropical en Río Hato, Panamá (en línea). Colombia Forestal 18(1): 105-115. Consultado 11 set. 2018. Disponible en http:// revistas.udistrital.edu.co/ojs/index.php/colfor/ article/view/7667/9880

Luoman, B; Mejia, A; Nuñez, L. 2002. Inventarios forestales para bosques latifoliados en América Central. Eds. Lorena Orozco, Cecilia Brumér. Turrialba, Costa Rica. Centro Agronómico Tropical de Investigación y Enseñanza (CATIE). 278 p.

Mahecha, L; Rosales, M; Molina, C y Molina, E. 2001. Experiencias en un sistema silvopastoril de Leucaena leucocephala - Cynodon plectostachyus -Prosopis juliflora en el Valle del Cauca, Colombia In Conferencia electrónica de la FAO sobre "Agroforestería para la producción animal en Latinoamérica" (en línea). Calí, Colombia, CIPAV. 325-336p. Consultado 22 abr. 2016. Disponible en http://www.fao.org/livestock/agap/frg/agrofor1/Mahech20.htm 
Miranda, J; Rusch, G; Casals, P; Declerck, F; Ibrahim, M; Casanoves, F y Jiménez, F. 2013. Efectos de los rasgos morfológicos y eco fisiológicos de árboles neo tropicales en la transferencia de agua y nutrientes al suelo. Agroforestería de las Américas 50: 69-75.

MOBOT (Missouri Botanical Garden). 2018. Flora de Nicaragua (en línea). Saint Louis, Missouri. Consultado 17 set. 2018. Disponible en http://www.tropicos.org/NameSearch.aspx?projectid $=7$

Mora, J; Espinal, M; López, L y Quezada, O. 2015. Caracterización del Bosque Seco Tropical Remanente en el Valle de Agalta, Honduras. Ceiba 53(1): 38-56.

Mostacedo, B y Fredericksen, T. 2000. Manual de Métodos Básicos de Muestreo y Análisis en Ecología Vegetal (en línea). Ed. D Nash. Santa Cruz, Bolivia. 92 p. Consultado 10 may. 2016. Disponible en http://www.bio-nica.info/biblioteca/mostacedo2000ecologiavegetal.pdf

Munch, L y Ángeles, E. 1990. Métodos y técnicas de investigación. 1ra edición. Trillas. México. $166 \mathrm{p}$.

Murrieta, E; Finegan, B; Delgado, D; Villalobos, R y Campos, J. 2007. Identificación y caracterización florística de bosques naturales en el Corredor Biológico Volcánica Central Talamanca, Costa Rica (en línea). Recursos Naturales y Ambiente (51-52): 57-68p. Consultado el: 15 de octubre del 2018. Consultado 10 oct. 2018. Disponible en http://orton.catie.ac.cr/ repdoc/A2158e/A2158e.pdf

Osorio, J. 2014. Efecto de la cobertura arbórea sobre la ganancia de peso y el desempeño reproductivo de vacas Brahman en trópico bajo. Tesis MSc. Medellín, Colombia. Universidad de Antioquia. $158 \mathrm{p}$.

Pérez, E. 2006. Caracterización de sistemas silvopastoriles y su contribución socioeconómica a productores ganaderos de Copán, Honduras. Tesis Mag. MSc. Turrialba, Costa Rica. CATIE. 138 p.

Pezo, D; Ibrahim, M. 1998. Sistemas Silvopastoriles. Ed. rev. 2da edición. Proyecto agroforestal CATIE/GTZ. Turrialba, Costa Rica. 276 p.
Salas, JB. 1993. Árboles de Nicaragua. Nicaragua. IRENA. 388 p.

Sánchez, D; Villanueva, C; Rusch, GM; Ibrahim, M y DeClerck, F. 2013. Estado del recurso arbóreo en fincas ganaderas y su contribución en la sostenibilidad de la producción en Rivas, Nicaragua. 1ra edición. Turrialba, Costa Rica. CATIE. 50 p.

Serrano, JR; Andrade, HJ y Mora-Delgado, J. 2014. Caracterización de la cobertura arbórea en un a pastura del trópico seco en Tolima, Colombia. Agronomía Mesoamericana 25(1): 99-110.

Souza de Abreu, M. 2002. Contribution of trees to the control of heat stress in dairy cows the financial viability of livestock farms in humid tropics. Tesis Ph.D. Turrialba, Costa Rica. CATIE. $166 \mathrm{p}$.

Tolentino, M. 2009. Sistemas Agro y Silvopastoriles en El Limón, Municipio de Paso de Ovejas, Veracruz, México (en línea). Tesis Mag. MSc. Veracruz, México. Instituto de Enseñanza e Investigación en Ciencias Agrícolas. 70 p. Consultado 22 abr. 2016. Disponible en http:// www.biblio.colpos.mx:8080/jspui/bitstream/ handle/10521/1664/Bautista Tolentino M MC Agroecosistemas Tropicales 2009.pd$\underline{\mathrm{f} \text { ? } \text { sequence }=1}$

Wojciechowski, M; Lavin, M; Sanderson, M. 2004. A phylogeny of legumes (Leguminosae) based on Analysis of the plastid matk gene resolves many Well-supported subclades within the family. American Journal of Botany 91(11): 1846-1862. Consultado 12 set. 2018. Disponible en https://onlinelibrary.wiley.com/doi/ epdf/10.3732/ajb.91.11.1846

Presentado: $14 / 06 / 2018$

Aceptado: 03/11/2018

Publicado en línea: 28/12/2018 